\title{
Compromiso y empleabilidad de los recién titulados de formación profesional. Conclusiones para un rediseño de la modalidad formativa.
}

\section{Engagement and employability of the student newly titled of vocational training. Conclusions for a redesign of the training modality.}

\author{
Francisco del Cerro Velázquez \\ Universidad de Murcia. Murcia. España \\ fcerro@um.es \\ Francisco Javier Ramón Cano \\ Universidad de Murcia. Murcia. España \\ jramon@um.es
}

\begin{abstract}
Resumen
En el artículo se observan y analizan las referencias bibliográficas relacionadas con los datos de desajuste entre la formación en competencias que han recibido en el sistema educativo los egresados de Formación Profesional y el empleo que han desarrollado en el mundo laboral. Posteriormente, se analiza el caso particular de los egresados de la Formación Profesional de la Región de Murcia. En concreto, se valora cómo percibe un titulado de Formación Profesional, como enseñanza apoyada en la tecnología, su proceso de inserción laboral a los seis meses siguientes a la finalización de su formación académica para afrontar un entorno de transformaciones empresariales e industriales. La muestra, facilitada por la Consejería competente en materia de educación, se compone de 1110 alumnos titulados en la Región de Murcia de Formación Profesional de grado medio y de grado superior de la promoción del curso académico 2013/14, seleccionados de forma aleatoria. En este caso, los resultados muestran un desajuste entre la formación en competencias del recién titulado de formación profesional y su posterior empleo, confirman las tendencias observadas en las referencias bibliográficas. Por tanto, en las conclusiones se propone reconsiderar medidas que aborden la superación de este desajuste.
\end{abstract}

Palabras clave: Formación Profesional, desajuste de competencias, empleo, compromiso.

\begin{abstract}
:
In the article we observe and analyze the bibliographical references related to the mismatch data between the training in skills that the graduates of Vocational Training have received in the educational system and the employment that has developed in the labor world. Subsequently, the particular case of the graduates of Vocational Training of the Region of Murcia is analyzed. Specifically, it is assessed how a titled in Professional Training perceives its process of labor insertion six months after the completion of its academic training, as teaching supported by technology, to face an environment of business and industrial changes. The sample facilitated by the Educational Regional Department, is composed of 1110 students graduated in the Region of Murcia Professional Training of middle grade and higher grade of the course academic 2013/14, randomly selected. In this case, the results show a mismatch between the training in skills of the newly students titled in Vocational Training and their subsequent employment, confirm the trends observed in the bibliographical references. Therefore, in the conclusions it is proposed to reconsider measures that address the overcoming of this mismatch.
\end{abstract}

Keywords: Vocational training, skills mismatch, employment, engagement. 


\section{Introducción}

Desde hace bastante tiempo, en España se han venido realizando por parte de instituciones autonómicas del país un creciente número de estudios e investigaciones sobre el proceso de inserción laboral de los titulados de Formación Profesional del sistema educativo donde se pone de manifiesto que dicho nivel, por término medio, suele ser alto (Pérez Esparrells y Rahona López, 2008). Los beneficios de obtener un título de Formación Profesional le supone al trabajador que tenga más probabilidades de mantener su empleo, de promocionar y, sobre todo, de mejorar su salario (Medina y Sanz, 2009), de hecho, en los países desarrollados la empleabilidad es siempre superior en la población de mayor nivel de estudios (OCDE, 2014).

En España, desde 2010 los únicos niveles formativos que han incrementado la contratación de forma continuada han sido los de Formación Profesional de grado medio y superior, así como, universitarios. Este hecho indica una tendencia de mayor presencia en el mercado de trabajo de niveles formativos con una cualificación específica de Formación Profesional y universitarios en detrimento de los niveles con titulaciones de carácter general como son Bachiller, ESO o niveles educativos inferiores (SEPE, 2015).

Los expertos estiman que hasta 2020 cerca de dos tercios del crecimiento en el empleo tendrá lugar en la categoría de técnicos titulados de Formación Profesional en grado medio y superior, poniendo en énfasis que la FP en España tiene pocos estudiantes, y que aumentan constantemente el número de jóvenes que no estudian ni trabajan llegando a tasas del 23\%, solo superado por Grecia OCDE (2014). El desarrollo de la economía actual requiere titulados muy especializados, con formación que no necesariamente debe ser superior a los dos años. Los objetivos fijados por la Unión Europea para el año 2020 recogen la necesidad de incrementar el nivel de formación y cualificación tanto de los jóvenes en edad escolar como de la población trabajadora, para lo que es necesario reforzar, modernizar y flexibilizar las enseñanzas de Formación Profesional. En el ámbito nacional, el Gobierno de la nación debe concebir la Formación Profesional como instrumento clave para avanzar hacia un nuevo modelo de crecimiento económico (COM, 2010).

En recientes resultados de las tasas de inserción laboral de alumnos titulados de Formación Profesional se observan que presentan algunas diferencias en los niveles de desagregación por familias profesionales, lo cual es consistente en los hallazgos de las investigaciones realizadas en este campo (Gobierno de Cantabria, 2013) y (LANBIDE, 2013).

Tradicionalmente, en España las titulaciones de Formación Profesional que se ofertan en la enseñanza reglada no están, en algunos casos, muy vinculadas a los sectores productivos locales y regional, ni obedecen siempre a estrategias de futuro en un mercado altamente competitivo, lo que induce un desajuste entre demanda y oferta de empleo (Monreal y otros, 2002). De hecho, el empleo perdido durante la reciente recesión económica lo ha sido mayoritariamente en ocupaciones de bajo nivel de capacitación. Entre 2007 y 2013 más de la mitad de los trabajadores que no habían superado la educación primaria perdieron su empleo. En cambio, el número de ocupados con estudios superiores se incrementó en un dos por ciento (Ley No 30, 2015).

Compromiso y empleabilidad de los recién titulados de formación profesional. Conclusiones para un rediseño de la modalidad formativa. Francisco del Cerro Velázquez y Francisco Javier Ramón Cano. 
Según Serrano y Soler (2014), en la próxima década las probabilidades de empleo se concentrarán en los jóvenes de entre 16 y 34 años más cualificados por su nivel de estudios, pero también por las competencias que hayan adquirido, por lo que es necesario poner en marcha iniciativas públicas y privadas mucho más potentes que las actuales para mejorar la empleabilidad de los jóvenes escasamente cualificados, que se enfrentan a elevados riesgos de exclusión laboral.

En España, existe un desajuste del nivel educativo requerido por la ocupación laboral y el requerido a los candidatos inscritos, es decir, los puestos de trabajo a los que podría aspirar un titulado de Formación Profesional en muchas ocasiones los ocupan (o pretenden ocupar) titulados universitarios (ESADE, 2013). Concretamente, en el año 2013, un 52\% de la oferta de vacantes publicadas por InfoJobs (Portal de ofertas de empleo en España) destinada a titulados de Formación Profesional de grado superior fue ocupado por estudiantes universitarios en un porcentaje que asciende hasta un 70\%. Otros informes de expertos, como el realizado por Améscoa (2014), establece que la Formación Profesional ha pasado a ser el "hermano mayor" de la inserción laboral y en el futuro, puede ser la formación con mayores garantías de empleabilidad.

No obstante, pese a los efectos positivos que puede conllevar la Formación Profesional, el análisis de políticas nacionales, llevado a cabo por (Cedefop, 2011), pone de manifiesto que la Formación Profesional todavía no goza del mismo prestigio que la educación general.

Actualmente, existen elevadas tasas de desempleo juvenil, algo preocupante en los países de la Unión Europea y en particular en España por lo que hay un creciente interés por alcanzar un mejor ajuste entre las competencias profesionales y los requerimientos del mercado de trabajo. Además, ligado a una Formación Profesional que en España está infravalorada OCDE (2014) se suscita en la sociedad un interés permanente por conocer, entre otros aspectos, cuáles son los resultados que obtienen en el mercado laboral las personas que han estudiado un ciclo formativo de Formación Profesional.

En diciembre del año 2014, el Servicio de Formación Profesional de la Región de Murcia recoge mediante encuesta telefónica los datos sobre aspectos de inserción laboral del alumnado que había finalizado recientemente los estudios de Formación Profesional de grado medio y de grado superior en el curso académico 2013/14, dando a conocer que 6 de cada 10 titulados había conseguido tener un empleo de alguna forma relacionado con su sector durante los últimos seis meses, quedando pendiente analizar otros aspectos interesantes de los datos recogidos.

\section{Objetivos del estudio}

Se pretende analizar las publicaciones o referencias actuales que relacionan competencias adquiridas en la enseñanza reglada de Formación Profesional y el empleo posteriormente desarrollado.

Por otro lado, en base a los datos facilitados por el Servicio de Formación Profesional de la Región de Murcia, este estudio centra su atención en el concepto de empleabilidad que tienen los propios titulados de Formación Profesional sobre su reciente experiencia de incorporación al mercado laboral contemplando las variables ajuste ocupacional y perspectiva laboral.

Compromiso y empleabilidad de los recién titulados de formación profesional. Conclusiones para un rediseño de la modalidad formativa. Francisco del Cerro Velázquez y Francisco Javier Ramón Cano. 
Concretamente, respecto al recién titulado de formación profesional, los objetivos específicos que se plantean son:

- Identificar el concepto de empleabilidad que tienen los propios alumnos.

- Conocer los factores de motivación y de compromiso que despierta dicho concepto de empleabilidad.

- Exponer las consecuencias prácticas para el rediseño de la formación recibida.

\section{Metodología}

En primer lugar, observaremos los datos publicados sobre el desajuste entre la formación en competencias que han recibido en el sistema educativo los egresados de Formación Profesional y el empleo que ha desarrollado en el mundo laboral. En este sentido, las publicaciones no son muy numerosas, sin embargo, son suficientes para evidenciar el desajuste antes indicado.

En segundo lugar, se expone la metodología de estudio y se presentan los resultados correspondientes a las preguntas de percepción de la encuesta: "Evaluación de la empleabilidad de los titulados de Formación Profesional" facilitados por la Consejería competente en materia de educación. La metodología propuesta servirá para valorar cómo percibe un titulado de Formación Profesional, como enseñanza apoyada en la tecnología, su proceso de inserción laboral a los seis meses siguientes a la finalización de su formación académica para afrontar un entorno de transformaciones empresariales e industriales.

\subsection{Observación del desajuste entre la Formación Profesional por competencias y el empleo}

Como citábamos anteriormente, la observación de los datos publicados nos evidencia la existencia en mayor o menor medida de un desajuste entre la Formación Profesional por competencias y el empleo, así, por ejemplo, en la Formación Profesional Vasca según los datos ofrecidos por Lanbide-Servicio de Empleo Vasco (2013) existe una proporción de 55 por cada 100 titulados que están trabajando en su sector o un 40 según el Ministerio de Empleo y Seguridad Social (2015). Otros estudios también reflejan esta circunstancia de manera que el desajuste ocupacional en la FP parece continuar extendiéndose, (Ibáñez, 2016); (cedefop, 2011) y (OIT, 2015).

\subsection{Empleabilidad de los egresados de la Formación Profesional de la Región de Murcia}

\subsubsection{Población y muestra}

La población objeto del estudio la integra el alumnado titulado en formación profesional de grado medio y de grado superior en el curso académico 2013/14 en la Región de Murcia. La muestra ya venía seleccionada previamente a través de procedimientos aleatorios simples con un tamaño de 1110 titulados (524 mujeres y 589 hombres). A posteriori se ha obtenido un error muestral inferior al 5\% que se considera aceptable para un nivel de confianza del $95 \%$ y poblaciones finitas ( $\mathrm{p}$ y q $=0,5$ ).

En cuanto al grado del ciclo formativo se obtiene que hay un equilibrio en los encuestados con un $50,7 \%$ de grado medio y $49,3 \%$ de grado superior. En términos

Compromiso y empleabilidad de los recién titulados de formación profesional. Conclusiones para un rediseño de la modalidad formativa. Francisco del Cerro Velázquez y Francisco Javier Ramón Cano. 
generales, el nivel de desagregación por familia profesional tiene representatividad tal y como se refleja en la tabla 1 donde viene la comparación porcentual del valor muestral y poblacional (este último valor estimado a través de la matrícula del último curso previa a la titulación).

\section{Tabla 1}

Datos de la muestra por familia profesional

\begin{tabular}{|c|c|c|c|}
\hline Familia Profesional & $\begin{array}{l}\text { Tamaño } \\
\text { Muestra }\end{array}$ & $\begin{array}{c}\text { Población } \\
\text { Porcentaje } \\
\text { titulados } \\
\text { (valor estimado) }\end{array}$ & $\begin{array}{c}\text { Muestra } \\
\text { Porcentaje } \\
\text { Titulados }\end{array}$ \\
\hline Actividades Físicas y Deportivas & 35 & $3,45 \%$ & $3,15 \%$ \\
\hline Administración y Gestión & 202 & $18,97 \%$ & $18,20 \%$ \\
\hline Agraria & 26 & $2,25 \%$ & $2,34 \%$ \\
\hline Artes Gráficas & 3 & $0,25 \%$ & $0,27 \%$ \\
\hline Comercio y Marketing & 26 & $3,64 \%$ & $2,34 \%$ \\
\hline Edificación y Obra Civil & 15 & $1,53 \%$ & $1,35 \%$ \\
\hline Electricidad y Electrónica & 99 & $8,18 \%$ & $8,92 \%$ \\
\hline Energía y Agua & 4 & $0,64 \%$ & $0,36 \%$ \\
\hline Hostelería y Turismo & 27 & $3,27 \%$ & $2,43 \%$ \\
\hline Imagen Personal & 45 & $3,25 \%$ & $4,05 \%$ \\
\hline Imagen y Sonido & 6 & $1,09 \%$ & $0,54 \%$ \\
\hline Industrias Alimentarias & 12 & $0,99 \%$ & $1,08 \%$ \\
\hline Informática y Comunicaciones & 78 & $6,87 \%$ & $7,03 \%$ \\
\hline Instalación y Mantenimiento & 31 & $2,61 \%$ & $2,79 \%$ \\
\hline Madera, Mueble y Corcho & 3 & $0,18 \%$ & $0,27 \%$ \\
\hline Marítimo-Pesquera & 15 & $0,74 \%$ & $1,35 \%$ \\
\hline Química & 21 & $1,99 \%$ & $1,89 \%$ \\
\hline Sanidad & 282 & $26,04 \%$ & $25,41 \%$ \\
\hline Servicios Socioculturales y a la Comunidad & 104 & $8,30 \%$ & $9,37 \%$ \\
\hline Transporte y Mantenimiento de Vehículos & 76 & $5,60 \%$ & $6,85 \%$ \\
\hline
\end{tabular}

La tabla 2 ofrece la caracterización de la muestra de estudio por centro de procedencia (público, concertado o privado):

Tabla 2

Caracterización de la muestra de estudio por tipo de centro de procedencia

\begin{tabular}{ccccc}
\hline Tipo de centro & $\begin{array}{c}\text { Informantes } \\
\text { Muestra }\end{array}$ & $\begin{array}{c}\text { Porcentaje } \\
\text { Muestra }\end{array}$ & $\begin{array}{c}\text { Porcentaje } \\
\text { Población }\end{array}$ \\
\hline \multirow{3}{*}{ Válidos } & Públicos & 871 & $78,5 \%$ & $81,86 \%$ \\
& Concertado & 153 & $13,8 \%$ & $12,21 \%$ \\
& Privado & 86 & $7,7 \%$ & $5,93 \%$ \\
\hline \hline
\end{tabular}

En términos generales, los datos permiten comprobar que la muestra sigue criterios de afijación proporcional tanto por familia profesional como por centro de procedencia a excepción de las familias profesionales de Artes Gráficas y de Madera, Mueble y Corcho donde se han obtenido un número muy bajo de informantes para su representatividad.

Compromiso y empleabilidad de los recién titulados de formación profesional. Conclusiones para un rediseño de la modalidad formativa. Francisco del Cerro Velázquez y Francisco Javier Ramón Cano. 


\subsubsection{Instrumento de recogida de datos}

En la tabla 3 se muestran las preguntas formuladas en la entrevista telefónica para recoger la opinión del alumnado:

\section{Tabla 3}

Cuestionario de inserción laboral de titulados de Formación Profesional en la Región de Murcia.

\section{CUESTIONARIO DE EVALUACIÓN DE LA OCUPACIÓN LABORAL DE LOS TITULADOS DE} FORMACIÓN PROFESIONAL

$$
\text { Ítem 1: ¿Has estado trabajando en los últimos seis meses? }
$$

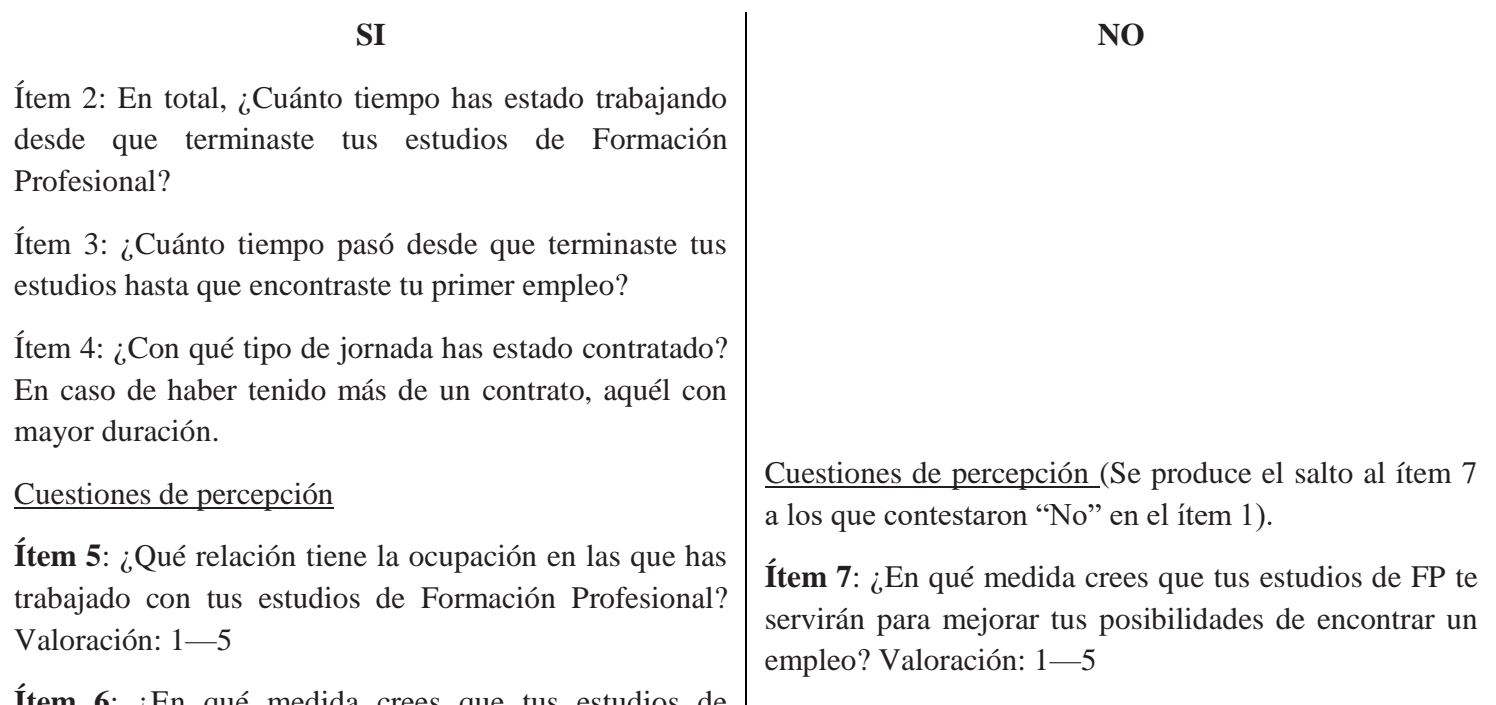
Formación Profesional te han servido para encontrar un empleo? Valoración: $1-5$

Ítem 8. ¿Tienes intención de seguir estudiando? SI/NO

Los ítems para cuestiones sobre percepción son los 5, 6, 7, los dos primeros son para quienes consiguieron encontrar un trabajo siguiendo un formato cerrado con una escala de tipo likert con cinco opciones de respuesta ( $1=$ nada, $2=$ poco, $3=$ algo, $4=$ bastante y $5=$ mucho). Mientras el ítem 7 está destinado a los que respondieron "No" a la pregunta del ítem 1 ¿Has estado trabajando en los últimos seis meses? con la misma escala likert .

Las variables a analizar en este trabajo son las siguientes:

Variable 1 Ajuste ocupacional: Ítem 5: ¿Qué relación tiene la ocupación en las que has trabajado con tus estudios de Formación Profesional?. Se pide al entrevistado que haya afirmado estar trabajando en el ítem 1 indique el grado de relación que tiene su título con la ocupación laboral desempeñada.

Variable 2 Perspectiva laboral: Ítem 6: ¿En qué medida crees que tus estudios de Formación Profesional te han servido para encontrar un empleo? Se pretende conocer la valoración que realiza el entrevistado sobre en qué medida sus estudios de Formación Profesional le van a servir para conseguir un empleo. Para quienes responden a que por el momento no han conseguido un empleo se plantea como pregunta alternativa el ítem 7:

Compromiso y empleabilidad de los recién titulados de formación profesional. Conclusiones para un rediseño de la modalidad formativa. Francisco del Cerro Velázquez y Francisco Javier Ramón Cano. 
¿En qué medida crees que tus estudios de FP te servirán para mejorar tus posibilidades de encontrar un empleo?

Para los instrumentos de recogida de datos en investigación educativa, se debe proceder a garantizar la validez y fiabilidad (Bisquerra, 2004). Para conocer su grado de fiabilidad se aplicó el alfa de Cronbach cuyo resultado para la dimensión que aquí se estudia sobre percepción tiene un valor de 0.763. Para autores como Huh, Delorme, and Reid (2006) el valor de alfa de Cronbach para una investigación exploratoria debe ser igual o mayor a 0,6 , mientras que para estudios confirmatorios debe estar comprendido entre 0,7 y 0,8 .

En cuanto a la validez del instrumento, se analizó la validez de contenido para los ítems 5 y 6, a través de un análisis factorial exploratorio. El análisis de la varianza total explicada tiene en cuenta un solo factor (autovalores mayores que 1) como se observa en la tabla 4.

\section{Tabla 4}

Varianza total explicada por los componentes. Método de extracción: Análisis de Componentes principales.

\begin{tabular}{c|c|c|c|c|c|c}
\hline \multirow{2}{*}{ Componente } & \multicolumn{4}{|c|}{ Autovalores iniciales } & \multicolumn{3}{c}{$\begin{array}{c}\text { Sumas de las saturaciones al cuadrado de } \\
\text { la extracción }\end{array}$} \\
\cline { 2 - 7 } & Total & $\begin{array}{l}\% \text { de la } \\
\text { varianza }\end{array}$ & $\begin{array}{c}\% \\
\text { acumulado }\end{array}$ & Total & $\begin{array}{l}\% \text { de la } \\
\text { varianza }\end{array}$ & $\begin{array}{c}\% \\
\text { acumulado }\end{array}$ \\
\hline 1 & 1,620 & 81,0 & 81,0 & 1,620 & 81,0 & 81,0 \\
\hline 2 &, 380 & 19,0 & 100 & & \\
\hline
\end{tabular}

Aunque el ajuste no fue perfecto, se demuestra una estructura unidimensional del cuestionario en nuestra población que a través de la extracción de un factor se podría explicar un $81 \%$ de la varianza total.

\subsubsection{Procedimiento de análisis de datos}

La metodología utilizada ha sido de carácter cuantitativo utilizando estadísticos descriptivos, pruebas técnicas de fiabilidad y validez de constructo (análisis factorial exploratorio). La perspectiva laboral en función del tipo de centro de procedencia se ha realizado mediante la prueba $\mathrm{H}$ de Kruskal-Wallis. Los datos obtenidos se analizaron mediante software estadístico SPSS v.17.0.

\section{Resultados del estudio}

\section{Ajuste ocupacional}

Centrándonos en los análisis de las fuentes observadas es evidente el desajuste ocupacional en la FP y además éste parece continua extendiéndose. En este sentido, para nuestra, como pasamos a comentar, ocurre algo similar una vez que analizamos la autopercepción de los egresados de la Formación Profesional.

A continuación, en la tabla 5, se presentan los resultados obtenidos relativos al ítem (5) sobre la percepción de los titulados que encontraron trabajo (en concreto 655 de los 1110 sujetos de la muestra), acerca del ajuste ocupacional.

Compromiso y empleabilidad de los recién titulados de formación profesional. Conclusiones para un rediseño de la modalidad formativa. Francisco del Cerro Velázquez y Francisco Javier Ramón Cano. 


\section{Tabla 5}

Descriptivos básicos de la autopercepción de la relación que tiene la ocupación con los estudios realizados de Formación Profesional

\begin{tabular}{llccccc}
\hline & & Nada (1) & Poco (2) & Algo (3) & Bastante(4) & Mucho (5) \\
\hline \multirow{2}{*}{ Grado Ciclo } & Ambos & $39,1 \%$ & $5,0 \%$ & $9,6 \%$ & $9,9 \%$ & $36,3 \%$ \\
\cline { 2 - 7 } & Medio & $40,7 \%$ & $3,3 \%$ & $9,4 \%$ & $8,8 \%$ & $37,8 \%$ \\
\cline { 2 - 6 } & Superior & $37,6 \%$ & $6,6 \%$ & $9,8 \%$ & $10,9 \%$ & $35,1 \%$ \\
\hline
\end{tabular}

Los resultados ofrecen cierta bipolaridad en la respuesta en los niveles 1 y 5 , por un lado hay un 46,2\% (36,3\% nivel 5 y 9,9\% nivel 4) de titulados que habiendo encontrado trabajo, afirman que la ocupación desempeñada bajo su punto de vista guardaba mucha relación con su formación académica y por el "lado opuesto" se tiene un 44,6\% de quienes afirman que poco o nada tiene que ver la formación recibida con la ocupación desempeñada (39,1\% de nivel 1 y $5 \%$ de nivel 2$)$; siendo en grado medio y grado superior resultados similares al del cómputo global. Si se abren los niveles tenemos cerca de un $61 \%$ de informantes que han conseguido un empleo que guarda alguna relación con los estudios realizados. Si se atiene al total de informantes de la muestra $(\mathrm{N}=1110)$ tanto hayan conseguido un trabajo o no, el resultado por familias profesionales sobre encontraron un empleo y además relacionado con su formación, distribuido porcentualmente por familias profesionales se expresa en la siguiente figura 1:

\begin{tabular}{|c|c|c|c|c|c|}
\hline $\begin{array}{r}\text { Actividades Físicas y } \\
\text { Deportivas }\end{array}$ & $37,1 \%$ & $14,3 \%$ & \multicolumn{3}{|c|}{$34,3 \%$} \\
\hline Administración y Gestión & $47,0 \%$ & \multicolumn{2}{|r|}{$23,3 \%$} & & $15,8 \%$ \\
\hline Agraria & $42,3 \%$ & \multicolumn{2}{|c|}{$19,2 \%$} & & $15,4 \%$ \\
\hline Artes Gráficas & $33,3 \%$ & \multicolumn{2}{|l|}{$33,3 \%$} & \multicolumn{2}{|c|}{$33,3 \%$} \\
\hline Comercio y Marketing & $30,8 \%$ & \multicolumn{2}{|l|}{$30,8 \%$} & \multicolumn{2}{|c|}{$26,9 \%$} \\
\hline Edificación y Obra Civil & $40,0 \%$ & \multicolumn{2}{|c|}{$26,7 \%$} & \multicolumn{2}{|c|}{$26,7 \%$} \\
\hline Electricidad y Electrónica & $39,0 \%$ & \multicolumn{2}{|l|}{$18,0 \%$} & & $14,0 \%$ \\
\hline Energía y Agua & $50,0 \%$ & \multicolumn{3}{|c|}{$25,0 \%$} & $25,0 \%$ \\
\hline Fabricación Mecánica & \multicolumn{2}{|l|}{$52,2 \%$} & \multicolumn{2}{|l|}{$21,7 \%$} & $13,0 \%$ \\
\hline Hostelería y Turismo & $11 \% \quad 18,5 \%$ & $14,8 \%$ & \multicolumn{3}{|c|}{$40,7 \%$} \\
\hline Imagen Personal & $33,3 \%$ & $24,4 \%$ & \multicolumn{3}{|c|}{$35,6 \%$} \\
\hline
\end{tabular}

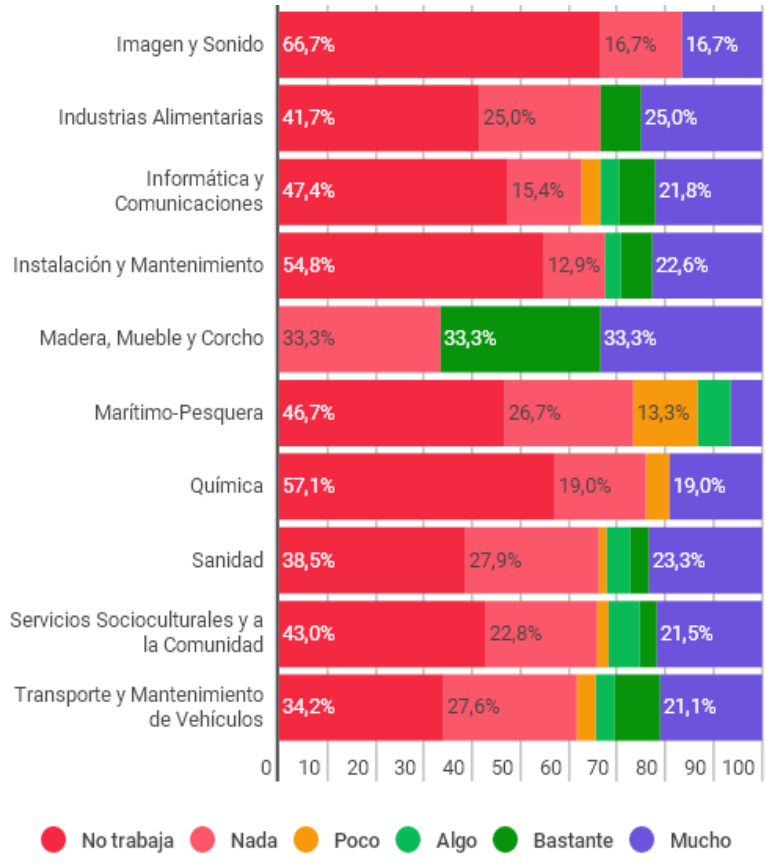

Figura 1. Relación percibida por el recién titulado de formación profesional entre la formación en competencias adquirida en educación y el puesto de trabajo

En cuanto a ajuste ocupacional, las que mejor comportamiento tienen son las de Hostelería y Turismo (71\%), Actividades Físicas y Deportivas (49\%), así como Imagen Personal (43\%) y Electricidad y Electrónica (43\%) (se deja al margen, por no ser representativas, las familias profesionales de Artes Gráficas (100\%) y Madera, Mueble y

Compromiso y empleabilidad de los recién titulados de formación profesional. Conclusiones para un rediseño de la modalidad formativa. Francisco del Cerro Velázquez y Francisco Javier Ramón Cano. 
Corcho (67\%)). Las familias profesionales con peor comportamiento en el ajuste ocupacional son las de Imagen y Sonido (16,7\%), Química (24\%), Fabricación Mecánica (27\%) y Administración y Gestión -se tiene dudas razonables para encuadrar en este grupo de desajuste ocupacional las familias profesionales Marítimo-Pesquera (27\%) y de Energía y Agua (25\%) por el número bajo de informantes que se dispone de las mismas-.

\section{Perspectiva laboral de los que encontraron trabajo}

Continuando el análisis descriptivo, en la figura 2 se encuentra por familia profesional los valores obtenidos para las respuestas sobre "perspectiva laboral", es decir, grado de autopercepción que tiene el recién titulado de los estudios logrados de Formación Profesional para ayudarle a encontrar un empleo (ítem 6):

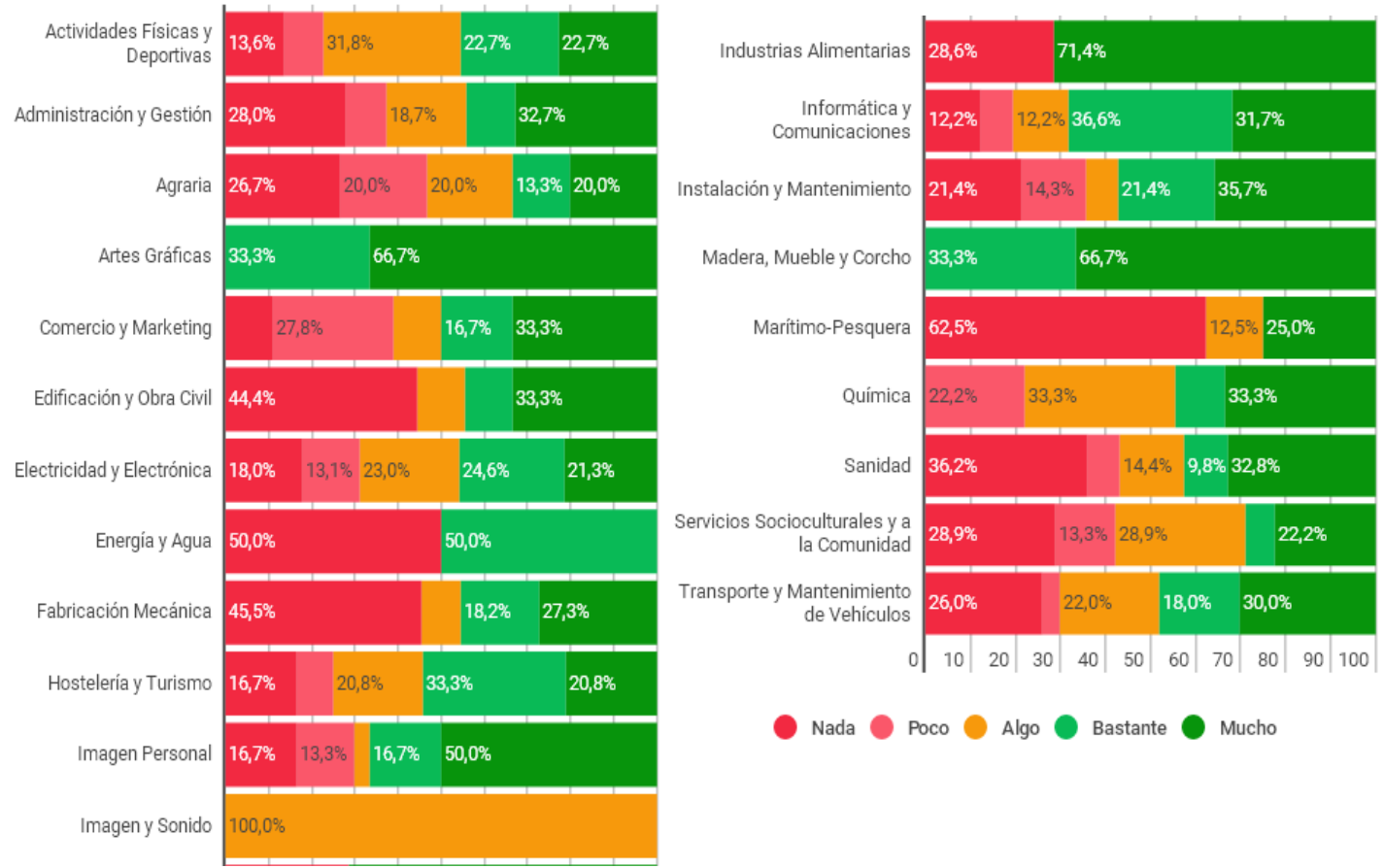

Figura 2. ¿En qué medida crees que tus estudios de Formación Profesional te han servido para encontrar un empleo?

Categorizando las respuestas en dos grupos:

- Las respuestas Nada, Poco y Algo $(1,2,3)$ supondrán un «nivel insuficiente» de la percepción de utilidad de los estudios realizados para desempeñar un puesto de trabajo.

- Las respuestas Bastante y Mucho (4,5) son consideradas suficientes para concluir que el alumno se siente satisfecho con los estudios realizados de cara a una inserción laboral.

Podemos afirmar, tal y como se observa, que los resultados en términos generales son positivos. Al ser desagregados por familia profesionales cabe reseñar negativamente los niveles de valoración insuficientes en las familias profesionales de Administración y Gestión (45\%), Fabricación Mecánica (45\%), Agraria (33\%), Marítimo-Pesquera (37,5\%), Química (40\%) e Imagen y Sonido (0\%).

Compromiso y empleabilidad de los recién titulados de formación profesional. Conclusiones para un rediseño de la modalidad formativa. Francisco del Cerro Velázquez y Francisco Javier Ramón Cano. 


\section{Perspectiva laboral de los que NO encontraron trabajo}

Para los titulados que no lograron un empleo se tiene una media del 40,1\% de informantes de la muestra que lo consideran satisfactorio (valores 4 y 5), en la indiferencia se sitúan un 35,8\% (valor 3), mientras que la valoración negativa (valores 1 y 2) se encuentra cerca de un $25 \%$ o lo que es lo mismo, 1 de cada 4 titulados de la muestra que no encontró trabajo en los primeros meses cree que su título no le podrá ayudar a encontrarlo.

Los resultados por familia profesional varían considerablemente (Figura 3). Así, las familias profesionales de carácter técnico (Electricidad y Electrónica, Informática y Comunicaciones o Agraria) son algunos ejemplos de mejores índices de satisfacción presentan.
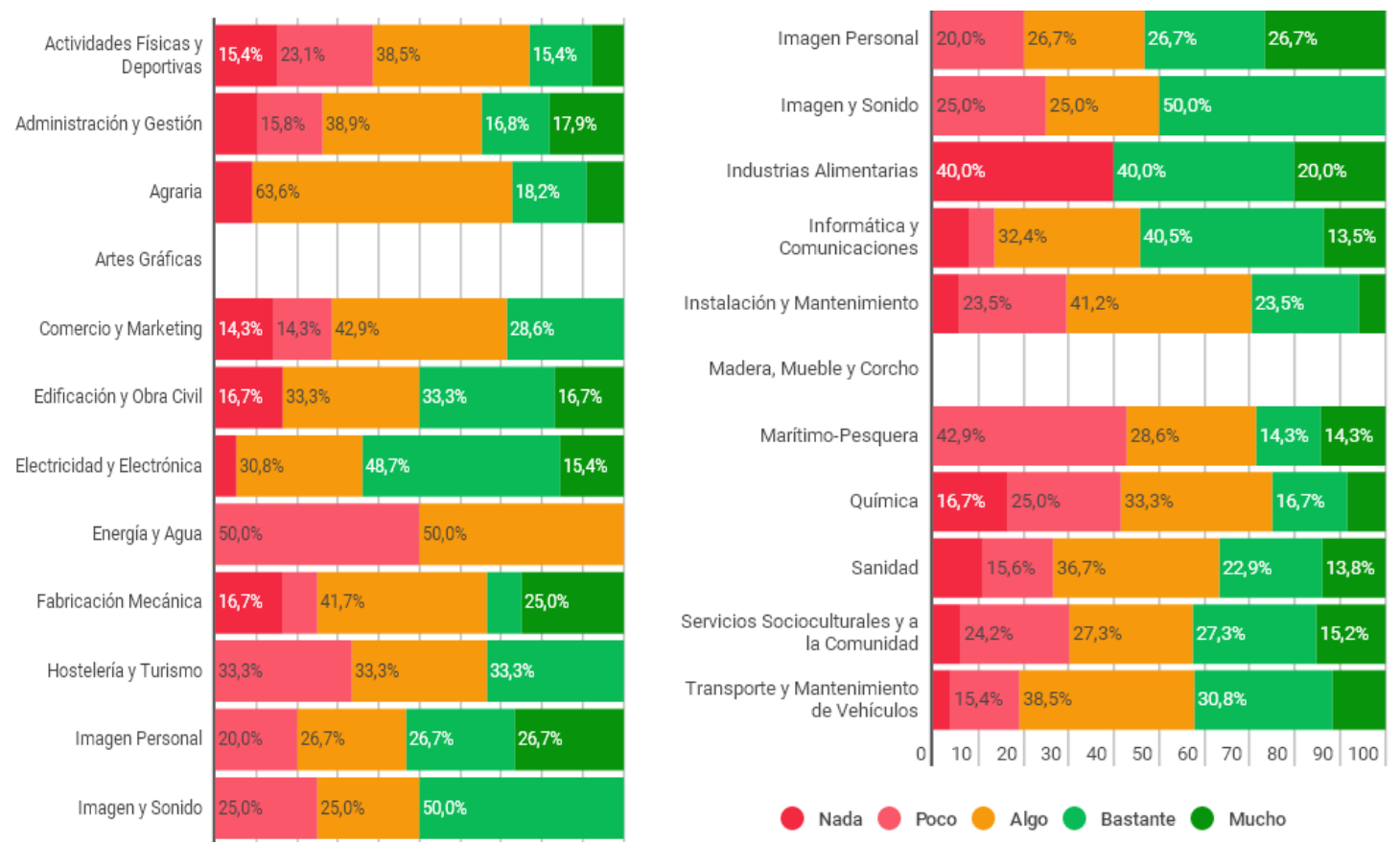

Figura 3. ¿En qué medida crees que tus estudios de FP te servirán para mejorar tus posibilidades de encontrar un empleo?

\section{Diferencias en las percepciones del alumnado en función del centro docente donde han realizado los estudios de formación profesional (privado, concertado, público).}

Tras la realización de la prueba de Kruskal-Wallis se encontró que había diferencias significativas para los ítems 6 (Sig. asintót. $=0,007 ; \mathrm{p}<0,05)$ y 7 (Sig. asintót. $=0,048 ; \mathrm{p}$ $<0,05)$ según el tipo de centro de procedencia del titulado de formación profesional: privado, público o concertado, tal y como se refleja en la siguiente tabla 6.

Compromiso y empleabilidad de los recién titulados de formación profesional. Conclusiones para un rediseño de la modalidad formativa. Francisco del Cerro Velázquez y Francisco Javier Ramón Cano. 


\section{Tabla 6.}

Resultado de la prueba de Kruskal-Wallis y rangos promedios de los ítems 6 y 7 en función del tipo de centro de procedencia

\begin{tabular}{|c|c|c|c|}
\hline \multirow{2}{*}{$\begin{array}{lc} & \begin{array}{l}\text { ¿En qué medida crees que tus estudios de Formación } \\
\text { Profesional te han servido para encontrar un empleo? }\end{array} \\
\text { Chi-cuadrado } & 9,853 \\
\end{array}$} & \multicolumn{3}{|c|}{$\begin{array}{l}\text { ¿En qué medida crees que tus } \\
\text { estudios de FP te servirán para } \\
\text { mejorar tus posibilidades de } \\
\text { encontrar un empleo? }\end{array}$} \\
\hline & \multicolumn{3}{|c|}{6,091} \\
\hline Gl & \multicolumn{3}{|c|}{2} \\
\hline Sig. asintót. & \multicolumn{3}{|c|}{,048 } \\
\hline \multicolumn{4}{|l|}{ Rangos promedios según el tipo de centro } \\
\hline & Tipo Centro & $\mathrm{N}$ & $\begin{array}{l}\text { Rango } \\
\text { promedio }\end{array}$ \\
\hline \multirow{4}{*}{$\begin{array}{l}\text { Ítem 6. ¿En qué medida crees que tus estudios de Formación Profesional te } \\
\text { han servido para encontrar un empleo? }\end{array}$} & Privado & 51 & 250,97 \\
\hline & Público & 519 & 333,44 \\
\hline & $\begin{array}{l}\text { Privado } \\
\text { concertado }\end{array}$ & 85 & 341,01 \\
\hline & Total & 655 & \\
\hline \multirow{4}{*}{$\begin{array}{l}\text { Ítem 7. ¿En qué medida crees que tus estudios de FP te servirán para mejorar } \\
\text { tus posibilidades de encontrar un empleo? }\end{array}$} & Privado & 35 & 200,64 \\
\hline & Público & 350 & 223,47 \\
\hline & $\begin{array}{l}\text { Privado } \\
\text { concertado }\end{array}$ & 68 & 258,72 \\
\hline & Total & 453 & \\
\hline
\end{tabular}

Se observa, en la tabla anterior, que los titulados que provienen de centros privados se muestran algo más pesimistas (rango promedio más bajo) con su formación como herramienta de ayuda para encontrar un empleo, así como, para mejorar las posibilidades de encontrarlo, respecto a los que proceden de centros docentes sostenidos con fondos públicos.

\section{Discusión y conclusiones}

Centrándonos en los análisis de datos, es posible afirmar que la percepción por el recién titulado de formación profesional es de cierto desajuste entre la formación en competencias que ha recibido en el sistema educativo y el empleo que ha desarrollado a los seis meses de finalizar su formación en el centro docente, en línea del desajuste al que hemos hecho referencia y que está sucediendo en mayor o menor medida en todo el territorio nacional.

En la Región de Murcia, las familias profesionales mejor posicionadas según los entrevistados en cuanto ajuste ocupacional han sido Hostelería y Turismo, Actividades Físicas y Deportivas, Imagen Personal y Electricidad y Electrónica. En el grupo de desajuste ocupacional cabe reseñar la familia profesional de Administración y Gestión por ser de amplia implantación en la Región de Murcia. Una de las posibles causas que ocasionan este nivel de desajuste en esta familia profesional, entre otras, podría estar en la existencia de una elevada proporción de graduados universitarios relacionados con dicha familia profesional que trabajan en ocupaciones de rango inferior al que les correspondería por su nivel educativo (Felgueroso y Jiménez-Martín, 2012); lo que hace que deriven a optar a otros sectores de empleos. Dichos autores y otros como Echeverría (2016) proponen para reducir el desajuste ocupacional en la formación profesional, entre otras medidas, la FP dual, que combina de forma simultánea la formación en la escuela con la formación en la empresa, importado del "modelo germánico", donde su éxito o

Compromiso y empleabilidad de los recién titulados de formación profesional. Conclusiones para un rediseño de la modalidad formativa. Francisco del Cerro Velázquez y Francisco Javier Ramón Cano. 
fracaso depende de las empresas de arraigada tradición local en la zona, que son sin duda el mejor escenario posible para que un desempleado de FP obtenga su primer empleo.

Preguntados por la perspectiva laboral, los recién titulados que encontraron trabajo muestran una valoración bastante buena de la formación académica recibida, considerada aceptable en términos generales en todas las familias profesionales. Las respuestas sobre utilidad del título para encontrar un futuro empleo realizado a quienes no encontraron trabajo dan como resultado que 1 de cada 4 titulados piensa que su título no le podrá ayudar a conseguir una ocupación laboral. Indagando en los resultados por el centro de procedencia se tiene que los titulados de centros privados se muestran ligeramente más pesimistas en las respuestas a perspectiva laboral que los titulados procedentes de centros sostenidos con fondos públicos, una de las posibles causas es la concentración de la oferta privada en determinadas áreas formativas como Sanidad, que a menudo están captando alumnos entre profesionales que tan solo buscan ampliar su formación.

En España, en los últimos años las políticas educativas se han esforzado en incrementar el número de estudiantes de Formación Profesional de grado medio y de grado superior, así entre el curso académico 2007/08 y el 2015/16 se han alcanzado incrementos del 68\%, pasando de 462.492 alumnos a 777.018 según los datos ofrecidos por la página web todofp.es del Ministerio de Educación, Cultura y Deporte. A nuestro entender este esfuerzo requiere medidas adicionales de actuación por parte de la Administración Educativa estatal y Autonómica para reducir el desajuste ocupacional en la enseñanza y por ende su encarecimiento.

Presentación del artículo: 6 de abril de 2017

Fecha de aprobación: 15 de junio de 2017

Fecha de publicación: 30 de junio de 2017

Del-Cerro-Velázquez, F. y Ramón Cano, F.J. (2017). Compromiso y empleabilidad de los recién titulados de formación profesional. Conclusiones para un rediseño de la modalidad formativa. RED. Revista de Educación a Distancia, 54. Consultado el (dd/mm/aaaa) en http://www.um.es/ead/red/

\section{FINANCIACIÓN}

El presente trabajo se inserta (utiliza los datos cedidos por el Servicio de Formación Profesional de la Región de Murcia) en el proyecto de investigación "Inserción laboral de titulados de Formación Profesional en la Región de Murcia -Curso 2013/2014-“, reconocido y financiado por Dirección General de Formación Profesional de la Región de Murcia.

\section{Bibliografía}

Améscoa, A. (2014). Propuestas desde el ámbito empresarial y estrategia de futuro para la Formación Profesional y el Empleo. [Versión electrónica en HTML]. Navarra: Servicio navarro de empleo. Fecha de consulta: 24 de marzo de 2017. En http://studylib.es/doc/4541498/propuestas-desde-el-ámbito-empresarial-y-estrategiade

Compromiso y empleabilidad de los recién titulados de formación profesional. Conclusiones para un rediseño de la modalidad formativa. Francisco del Cerro Velázquez y Francisco Javier Ramón Cano. 
Bisquerra, R. (Ed.). (2004). Metodología de la investigación educativa. Madrid: La Muralla.

Centro Europeo para el Desarrollo de la Formación Profesional (2011). A pesar de sus numerosos beneficios, la Formación Profesional carece de reconocimiento. (Nota Informativa). Salónica (Grecia): Cedefop. Fecha de consulta: 24 de marzo de 2017. En https://www.sepe.es/contenidos/personas/formacion/refernet/pdf/mayo2011.pdf

COM (2010). Europa 2020: Una estrategia para un crecimiento inteligente, sostenible $e$ integrador. [Versión electrónica en PDF]. Bruselas: Comisión Europea. Fecha de consulta: 2 de marzo de 2017. En

http://eur-lex.europa.eu/LexUriServ/LexUriServ.do?uri=COM:2010:2020:FIN:ES:PDF

Echeverría Samanes, B. (2016). Transferencia del Sistema de FP Dual a España. Revista de Investigación Educativa. 34(2), 295-314 Fecha de consulta: 2 de marizo de 2017. DOI: http://dx.doi.org/10.6018/rie.34.2.249341

ESADE (2013). Informe económico 14. [Versión electrónica en PDF]. Barcelona: ESADE (Escuela Superior de Administración y Dirección de Empresas). Fecha de consulta: 2 de marzo de 2017. En http://itemsweb.esade.edu/biblioteca/archivo/informeeconomicoenero2013.pdf

Felgueroso, F. y Jiménez-Martínez, S. (2012). Formación Profesional: No debemos tropezar dos veces en la misma piedra. Fecha de consulta: 15 de marzo de 2017. En https://studylib.es/doc/5237757/formación-profesional--no-debemos-tropezar-dosveces-en-la

García, E., Gil, J. y Rodríguez, G. (2000). Análisis factorial. Madrid: La Muralla.

Gobierno de Cantabria (2013). Informe de Inserción Laboral 2013-2014. Titulados de Formación Profesional en centros sostenidos con fondos públicos.

Huh, J.; DeLorme, D.E.; and Reid, L.N. (2006). Perceived third-person effects and consumer attitudes on prevetting and banning DTC advertising. Journal of Consumer Affairs, 40(1):90-116.

Ibañez, M. J. (4 de enero de 2016). Solo tres de cada 100 graduados en FP logran un empleo en su especialidad. El Periódico. Fecha de consulta: 15 de marzo de 2017. En http://www.elperiodico.com/

Lanbide-Servicio Vasco de Empleo (2013). Inserción laboral de los titulados en Formación Profesional en 2013. Versión electrónica en HTML]. País Vasco: Servicio vasco de empleo. Fecha de consulta: 24 de marzo de 2017. En http://www.lanbide.euskadi.eus/estadistica/insercion-laboral-de-los-titulados-enformacion-profesional-en-2013/y94-estadist/es/

Ley Orgánica 2/2006, de 3 de mayo, de Educación. Boletín Oficial del Estado, 106, 4 de mayo de 2006.

Compromiso y empleabilidad de los recién titulados de formación profesional. Conclusiones para un rediseño de la modalidad formativa. Francisco del Cerro Velázquez y Francisco Javier Ramón Cano. 
Ley 30/2015, de 9 de septiembre, por la que se regula el Sistema de Formación Profesional para el empleo en el ámbito laboral. Boletín Oficial del Estado, 217, 10 de septiembre.

Medina, O. y Sanz, F. (2009). Los sistemas de reconocimiento y acreditación de los aprendizajes no formales e informales: referencias históricas, funciones socioeducativas y perspectiva teórica. Revista de Educación, 348, 253-281.

Ministerio de Empleo y Seguridad Social (2015). Reforma del Sistema de Formación Profesional para el empleo en el ámbito laboral. Versión electrónica en PDF]. Madrid: Ministerio de Empleo y Seguridad Social.

Monreal, J. y otros (2002). La Formación Profesional y su adecuación al mercado laboral. [Versión electrónica en PDF]. España: Compobell, S.L. Fecha de consulta: 6 de marzo de 2017. En: http://docplayer.es/16161032-La-formacion-profesional-y-suadecuacion-al-mercado-laboral.html

Organización para la Cooperación y Desarrollo Económico (2014). Panorama de la educación. Indicadores de la OCDE 2014. Versión electrónica en HTML]. Madrid: Instituto Nacional de Evaluación Educativa (inee). Fecha de consulta: 6 de marzo de 2017. En: http://www.mecd.gob.es/inee/Ultimos_informes/Panorama-de-laEducacion-2014.html

Organización Internacional del Trabajo (2013). El desajuste entre oferta y demanda de calificaciones afecta la creación de empleo. Versión electrónica en HTML]. Madrid. Fecha de consulta: 24 de marzo de 2017. En: http:/www.ilo.org/global/about-theilo/newsroom/news/WCMS_203945/lang--es/index.htm

OECD (2014). Skills beyond School: Synthesis Report, OECD Reviews of Vocational Education and Training. Paris: OECD Publishing. Fecha de consulta: 15 de marzo de 2017. DOI: http://dx.doi.org/10.1787/9789264214682-en

Pérez, M.C., and Rahona, M. (2009). La calidad de Formación Profesional y sus implicaciones para el mercado de trabajo. Revista del Instituto de Estudios Económicos, ISSN 0210-9565, Nº 2, 2009 (Ejemplar dedicado a: Los nuevos retos de la Formación Profesional), págs. 71-102

Pérez de las Vacas, C. (2015). Inserción Laboral de universitarios desde la perspectiva psicosocial. (Tesis doctoral). Universidad de Extremadura, España. Fecha de consulta: 15 de marzo de 2017. En

http://dehesa.unex.es/bitstream/handle/10662/4040/TDUEX_2016_Perez\%20de\%201 as\%20Vacas_Aparicio.pdf?sequence $=1$

Servicio Público de Empleo Estatal (2015). Observatorio de las Ocupaciones: 2015 Informe del Mercado de Trabajo Estatal. [Versión electrónica en PDF]. Madrid: SEPE. Fecha de consulta: 17 de marzo de 2017. En https://www.sepe.es/contenidos/que_es_el_sepe/publicaciones/pdf/pdf_mercado_trab ajo/imt2015_datos2014_estatal_general.pdf

Compromiso y empleabilidad de los recién titulados de formación profesional. Conclusiones para un rediseño de la modalidad formativa. Francisco del Cerro Velázquez y Francisco Javier Ramón Cano. 
Serrano y Soler (2014) Informe Fundación BBVA-Ivie: La formación y el empleo de los jóvenes españoles. Trayectoria reciente y escenarios futuros (versión Mayo 2015). Fecha de consulta: 2 de marzo de 2017. En http://www.fbbva.es/TLFU/tlfu/esp/noticias/fichanoticia/index.jsp?codigo=1424 